\section{Microcomputer-resident comprehensive statistical analysis}

\section{GREGORY J. BUHYOFF, HARALD M. RAUSCHER, R. BRUCE HULL IV, and KEVIN KILLEEN Virginia Polytechnic Institute and State University Blacksburg, Virginia 24061}

A comprehensive statistical processing system (SPS Version 2.0) for the Z80-based microcomputer is described. The package is structured in a format similar to the main frame SPSS package (Nie, Hull, Jenkins, Steinbrenner, \& Bent, 1975) and includes many of its file handling capabilities.

\section{Hardware and Firmware}

SPS programs were written for a Tandy Radio Shack Model I computer that utilizes the Z80 microprocessor and Microsoft BASIC. System configuration must be a minimum $32 \mathrm{~K}$ RAM, at least one disk drive, and the TRSDOS or NEWDOS ${ }^{1}$ operating system. Hard-copy output can be enabled via operating system utilities or insertion of program statements to change PRINT to LPRINT (Buhyoff \& Hull, 1980). Either serial or parallel printers may be used.

\section{Program Algorithms and Outputs}

SPS consists of 32 programs written in BASIC No COMMON or CHAIN commands are available in the the BASIC dialect utilized. The programs use the disk to store intermediate results and partial data files. The system is "user friendly," in that it is interactive and self-documenting and, once entered, requires only a series of option selections by the user.

File Creation and Handling. This module permits the creation of new data files and modification of existing ones. All file output is to disk in sequential form. Sequential rather than random format is used to speed input and output. Data are built into an $\mathrm{X}_{\mathrm{ij}}$ matrix, where $\mathrm{i}=$ number of observations and $\mathrm{j}=$ number of variables. Coding and data input follow the same logic used in creating input for card images. Missing data can be specially coded. A single-file matrix structure is then used by all SPS routines. Variable names are input using any combination of numeric or alphanumeric characters. Data entry is at the keyboard, with an option to exit the data entry mode at any time and save the partial file input. Files can be reviewed and corrected (including variable names), listed on a printer, truncated, or expanded by deleting or adding observations or variables.

A routine permits select variables to be "stripped"

H. M. Rauscher is Research Forester, USDA Forest Service, Northern Hardwoods Lab, Marquette, Michigan. off a file matrix and saved as a new, smaller file. Options also exist for transposing the $\mathrm{X}_{\mathrm{ij}}$ matrix to an $\mathrm{X}_{\mathrm{ji}}$ structure, and, transforming variable values via the use of additive and multiplicative constants, logs to any base, exponents, square root, reciprocal, and the standardized $\mathrm{z}$ transform. Data may be sorted on any variable code or range of codes and the truncated matrix written as a new file. Recoding of variable values is supported, as well as the formation of new variable indexes, through any linear or multiplicative combination of variable values. Finally, data matrices can be rank ordered, with a provision for breaking ties by the average-of-the-sums method.

One of the most important file handling options supported by SPS is that of file merging. Since SPS algorithms rely on in-memory routines and sequential output format, data file sizes are limited by RAM constraints. However, by breaking the $\mathrm{X}_{\mathrm{ij}}$ matrix into several smaller $X_{i k}$ (where $k<j$ ) data files, it is possible to store larger data sets on disk than available memory will allow. This is done by stripping and merging to create the $X_{i k}$ structure needed for any statistical algorithm. A $48 \mathrm{~K}$ system will hold, in memory, an SPS $X_{i j}$ file that can range from $i=2,600, j=2$ to, for example, $i=360$, $j=20$. However, by stripping variables of interest from several smaller files and merging them, any specific $X_{i k}$ structure can be written and used by SPS statistical programs. For example, an $\mathrm{X}_{360,60}$ matrix may be stored as three $X_{360,20}$ matrices. Most SPS statistical algorithms, other than multiple regression, only require an $X_{i, 2}$ matrix.

Descriptive Statistics and Plotting. Standard descriptive statistics are output, including the min, max, range, median, mean, variance, mode, moments about the mean and coefficients of skewness and kurtosis. Histogranis (output graphically to the CRT) and frequency distributions can be computed and plotted for up to 36 intervals. XY scatterplots displayed on the CRT are also supported. A multiple-variable plotting algorithm permits the simultaneous plotting of up to six $Y$ variables against a single $X$. This output is constrained on a printer and allows user-defined labels and axes, specification of plotting ranges, and horizontal and vertical line control.

Mean Tests. With the system, $t$ tests can be computed, including the approximation to the $t$ for cases of unequal variances. Homogeneity of variances is also tested using the F-max test. One-way ANOVA (fixedeffects) models can be computed along with tests for equal-variance terms and a posteriori multiple comparisons utilizing Gabriel's sum-of-squares simultaneous test procedure, which is an exact test for both equal and unequal treatment sample sizes.

Cross-Tabulations. This module permits partitioning a data file into contingency tables ranging from 2 by 2 
to $\mathrm{R}$ by $\mathrm{C}$, where $\mathrm{R}$ and $\mathrm{C}$ are less than or equal to 10 . Variables may be partitioned on either integer codes or ranges of real numbers. Output statistics are chi square, phi, Cramer's V, and the contingency coefficient.

Test Distributions. The $t, F$, and $\chi^{2}$ distributions can be used to obtain attained levels of significance (p) by inputting degrees of freedom and critical test statistic values from SPS statistical tests.

Monte Carlo Distributions. This algorithm generates SPS data files containing random observations that conform to the uniform, normal, or log-normal distributions. Either real or integer values may be specified, as well as the distributional range and desired parameter values. These simulated data may be written on disk.

Correlation. Both parametric and nonparametric correlation analyses are supported. Pearson's $r$ with accompanying $t$ tests of $\mathrm{H}_{0}: \mathrm{r}=0$ and partial correlations based on the $\mathrm{r}$ matrix can be computed for any subset of a specified data file. Also, the nonparametric Spearman's rho, Kendall's $W$, and the point biserial $r$ are part of SPS. The Pearson's $r$ routine also permits a resultant correlation matrix to be written to disk for other future analyses, including the multivariate techniques discussed below.

Regression Analyses. Simple and multiple linearregression modeling is supported by three separate algorithms. First, simple linear and linearized log, power, and exponential curve fits can be output. An ANOVA table and summary sum-of-squares values are printed along with the appropriate $F$ test, confidence intervals for the slopes, $\mathrm{r}^{2}$, and standard error of estimates. The $\hat{\mathrm{Y}}$ function may be plotted on the CRT according to user-specified axes parameters. Residuals are computed and plotted in normalized form, and the option exists for writing them on disk for further analyses. Second, multiple linear-regression models may be computed for up to 10 independent variables. The routine utilizes the Gaussian elimination method (Seber, 1977). ANOVA statistics are output, as well as $R^{2}$, adjusted $R^{2}$, Durbin Watson statistic, variance-covariance matrix of the coefficients, and a normalized residual plot. Residuals may, again, be written to a disk file.

Finally, a general linear-regression modeling routine is available. This routine is similar to the one employed in SAS and provides comparable results (Barr, Goodnight, Sall, \& Helwig, 1976). The program solves for general linear models of the form $y=b_{0}+b_{1} \phi_{1}+b_{2} \phi_{2}+\ldots$ $+b_{k} \phi_{k}$, where $\phi_{k}=f\left(X_{1}, X_{2}, \ldots, X_{k}\right)$. The singularvalue decomposition algorithm of Golub and Reinsch (1970) was used as the basis for the solution. While this algorithm is not the most time efficient, it is quite accurate (Forsythe, Malcolm, \& Moler, 1977). Up to 9 independent variables may be used when an intercept is specified; otherwise, 10 may be used. An ANOVA table, confidence intervals for the coefficient estimates, indexes of collinearity, normalized residual plots, the
Durbin Watson statistic, and standardized coefficients can all be computed. Confidence bands about the $\hat{Y}$ values and residuals may all be plotted on a printer using a variation of the multiple-variable plot routine cited above.

Multivariate Analyses. Factor, principal component, and image analysis programs are available in SPS. The input correlation matrices may be entered at the keyboard or input from a disk file created by the Pearson's $r$ program. The maximum correlation matrix size is 50 by 50 . The algorithm used to determine the characteristic roots and vectors is the Householder/Ortega/ Wilkinson method (Harman, 1976). The number of iterations may be specified, and at each new pass, new communality estimates are calculated from the resulting factor loading matrix and inserted into the original correlation matrix. Either the number of factors to be extracted or the minimum eigen value may be specified as termination constraints.

The factor loading matrix, eigen values, several estimates for proportion of variance accounted for by each factor, and the cumulative variance accounted for are the standard output for both principal components and factor analysis. In addition, the antiimage covariance matrix and the image covariance matrix are displayed when the image analysis option is selected.

Finally, routines are incorporated to compute both component and factor scores. The factor-score option uses a regression method for generation of the final scores.

\section{Computation Time Efficiencies}

Examples of approximate computation/run times of several of the highly iterative algorithms employed in SPS are given below.

(1) A 22 by 22 correlation matrix with 24 observations per variable vector was computed in $6.5 \mathrm{~min}$. (2) Approximately $5.25 \mathrm{~min}$ was needed to compute the coefficient estimates, test statistics, and ANOVA for a multiple linear-regression model based on an $X_{200,6}$ matrix. (3) Rank ordering a 30-element vector with 15 tied values requires approximately $50 \mathrm{sec}$ of CPU time. (4) A 30 by 30 correlation matrix was factor analyzed in $2 \mathrm{~h}$. Multivariate techniques are the most timeconsuming, and this time can increase exponentially with the size of the data matrix.

\section{Future Developments}

Currently, a general linear model ANOVA, robust regression, and nonparametric median tests are under development.

\section{Availability}

SPS Version 2.0 is available from Gregory J. Buhyoff, Department of Forestry, School of Forestry and Wildlife Resources, Virginia Polytechnic Institute and State 
University, Blacksburg. Virginia 24061. Ready-to-run program requests should include $\$ 5$ for printing costs of the 80-page users' manual and two blank 5.25 -in. softsectored floppy disks. Source code in the form of listings is also available. Users will be informed of changes and additions.

\section{REFERENCES}

Barr, A. J., Goodnicilit, J. H., Sall, J. D., \& Helwig, J. T $A$ users guide 10 SAS. 76. Raleigh, N.C: SAS Institute. Inc., 1976.

Bunyofr, G. J.. \& Hut., R. B.. IV. Computation of law of comparative judgment scales on a microcomputer. Behavior Research Methods \& Instrumentation, 1980, 12, 465.

Forsythe, G. E.. Malcom, M. A., \& Moler, C. B. Computer methods for mathematical computations. Englewood Cliffs, N.J: Prentice-Hall, 1977.
Golub, G. H., \& Reinsch, C. Singular value decomposition and least squares solutions. Numerical Mathematics, 1970, 14, 403-420.

Harman, H. H. Modern factor analysis. Chicago: University of Chicago Press, 1976.

Nie, N. H., Huli, H. C., Jenkins, J. G., Steinbrenner, K., \& BENT. D. H. SPSS: Statistical package for the social sciences (2nd ed.). New York: McGraw-Hill, 1975.

Seber, G. A. F. Linear regression analysis. New York: Wiley, 1977.

\section{NOTE}

1. TRSDOS is a trademark of Radio Shack, and NEWDOS is a trademark of Apparat, Inc., Denver, Colorado.

(Received for publication September 24, 1980; accepted October 10, 1980.) 